\title{
Point Symmetries of Controlled Systems and Their Applications
}

\author{
Victor I. LEHEN'KYJ \\ Kyïv Institute of Air Force, \\ 30 Povitrof lotsky Avenue, Kyïv, 252186, Ukraïna
}

\begin{abstract}
A problem of finding point symmetries of controlled systems is discussed, basic theorems and algorithms are formulated. The application to some problems of flight dynamics is suggested.
\end{abstract}

It is well known that the concept of point symmetries of a system of ordinary differential equations can be formulated in two ways:

1) the symmetry transfers solutions of system (1) into ones,

2) the symmetry preserves the form of system (1):

$$
\dot{x}^{i}=f^{i}(t, x), \quad i=\overline{1, n} .
$$

In contrast to the latter, dynamic systems with control are characterized by presence of some free functions in right-hand part of differential equations of the form (2), control actions $u$ :

$$
\dot{x}^{i}=f^{i}(t, x, u(\cdot)), \quad i=\overline{1, n} .
$$

The methodological problem in the development of group analysis of these systems is in the necessity of "a priori" choosing the principle of control, i.e., the form of the function $u$. For example, if $u=u(t)$, we speak about program control, at $u=u(t, x)$ about feedback control, at $u=u(t, x, \dot{x})$ about derivative control, etc.

Accordingly, the representations of solutions of system (2) are different. The present research is based on the concept of feedback control. Therefore we proceed from system (2) to the equivalent equation in first-order partial derivatives

$$
F=X_{0} S=S_{t}+f^{i}(t, x, u) S_{x^{i}}=0,
$$

where $X_{0}=\partial_{t}+f^{i} \partial_{x^{i}}$ is a vector field associated with system (2), and solutions of (3) are formed by the set of pairs of functions $(u(t, x), S(t, x))$.

We shall consider only controlled systems, when for equation (3) there exist no such solutions $S(t, x)$ which satisfy equation (3) for all functions $u(t, x)$ :

$$
\nexists \quad S(t, x): \quad X_{0} S=0 \quad \forall u(t, x) .
$$

The equation (3) has special properties:

Copyright (c) 1997 by Mathematical Ukraina Publisher. All rights of reproduction in any form reserved. 
- equation (3) is underdetermined, as it contains some dependent variables;

- control actions enter the equation functionally (algebraically);

- functions $f^{i}$ do not depend on $S$.

We shall consider symmetries of equation (3) in the class of operators of the following form:

$$
X=\tau(t, x, u, S) \partial_{t}+\xi^{i}(t, x, u, S) \partial_{x^{i}}+\varphi(t, x, u, S) \partial_{u}+\eta(t, x, u, S) \partial_{S}
$$

The standard condition of the symmetry

$$
\left.\underset{(1)}{X} F\right|_{F=0}=0
$$

gives us the system of determining equations

$$
\begin{aligned}
& X_{0} \eta=0, \quad U \eta=0, \quad\left(U=\partial_{u}\right), \\
& U \xi^{i}-f^{i} U \tau=0, \quad i=\overline{1, n}, \\
& X f^{i}+f^{i} X_{0} \tau-X_{0} \xi^{i}=0 .
\end{aligned}
$$

Due to the above-stated features and assumption about quite a controllability, the analysis of system (7)-(9) can be conducted in more detail.

From conditions (4) and (7) it follows that $\eta=\eta(s)$ and we automatically obtain the following statement:

Proposition 1 If equation (2) admits an operator of symmetry of form (10)

$$
X=\eta(t, x) \partial_{S}
$$

system (2) is non-controllable.

The next object of our analysis is the system (8). This system is also underdetermined, since it consists of $\mathrm{n}$ equations and $(n+1)$ unknown functions. Systems of similar forms are not well investigated. The following approach is suggested. Due to linearity of system (8), its general solution can be written in the form of a sum of a trivial general solution of the uniform equation $\left(\hat{\xi}^{i}(t, x)\right.$ are arbitrary functions) and a partial solution of the nonuniform equation

$$
\xi=\hat{\xi}^{i}(t, x)+\tilde{\xi}^{i}(t, x, u) .
$$

The main problem is of searching for a partial solution. The following result is formulated. 
Theorem 1 In the regular case (rank $\left\|U^{j} f i\right\|=n, j=\overline{1, n}$ ), a partial solution of system (8) has the form (12)

$$
\begin{aligned}
\tau & =\sigma^{(n)}+\sum_{k=0}^{n-1} A_{k} \sigma^{(k)}, \quad\left(\sigma^{(k)}=U^{k} \sigma\right), \\
\xi^{i} & =f^{i} \sigma^{(n)}+\sum_{k=0}^{n-1} B_{k}^{i} \sigma^{(k)}
\end{aligned}
$$

where $\sigma=\sigma(t, x, u, S)$ is an arbitrary function, and for determination of coefficients $\left(A^{i}, B_{k}^{i}\right)$ it is necessary to execute the following algorithm:

1) To solve the linear system of equations (13):

$$
W Y=V,
$$

where $W=\left\|U^{j} f^{i}\right\|, \quad Y=\left\|Y^{j}\right\|, \quad V=\left\|(-1)^{n+1} U^{n+1} f^{i}\right\|$;

2) To find $A_{l}$ by formula (14):

$$
A_{i}=\sum_{k=i}^{n-1}(-1)^{k}\left(\begin{array}{c}
k \\
i
\end{array}\right) U^{k-i} Y_{k}, \quad i=\overline{0, n-1} ;
$$

3) To determine the coefficients $B_{k}^{i}$ by recursion formulae (15), (16)

$$
\begin{aligned}
& B_{n-1}^{i}=f^{i} A_{n-1}-U f^{i}, \\
& B_{k}^{i}=-U B_{k+1}^{i}+f^{i}\left(A_{k}+U A_{k+1}\right), \quad k=\overline{0, n-2} .
\end{aligned}
$$

Theorem 2 When the controlled system (2) has $r$ control actions $\left(u_{1}, u_{2}, \cdots, u_{r}\right)$ and each one is essential, coefficients $\left(\tau, \xi^{i}\right)$ do not depend on $u: \tau_{u}=\xi_{u}^{i}=0$.

Because the functions $f^{i}$ do not depend on $S$, the following statement holds true.

Theorem 3 If $X$ is an operator of symmetry of equation (3), then the operator $\hat{X}=$ $\left[\partial_{S}, X\right]$ is also an operator of symmetry.

Thus, equations (7) and (8) have the constructive solution and it remains only to solve the system (9).

If we search for operators of symmetry in more narrow classes of operators of the following form

$$
Y=\tau(t, x) \partial_{t}+\xi^{i}(t, x) \partial_{x^{i}},
$$

we have the additional opportunity for decomposition of system (8), (9) on control actions. It permits to prove the following statement.

Theorem 4 The invariance algebra admitted by the controlled system (2) in the class of operators (17) has the finite dimension ( $\operatorname{dim} Y \leq n+2)$. If system (2) admits an $(n+2)$-dimensional invariance algebra, then there exists the morfism $\hat{t}=\hat{t}(t)$ for which the algebra $Y$ includes an $n$-dimensional ideal $Y_{1}=\xi^{i}(\hat{t}, x) \partial_{x^{i}}$ and 2-dimensional quotient algebra $Y_{2}=\left\{\partial_{\hat{t}}, \hat{t} \partial_{\hat{t}}+\mu^{i}(x) \partial_{x^{i}}\right\}$. 
This theorem generalizes the result of G.Yakovenko known previously.

Let us consider some examples.

Example 1 The invariance algebra of the system

$$
\dot{x}^{1}=-u^{2}, \quad \dot{x}^{2}=u
$$

is infinite:

$$
\begin{aligned}
X= & \alpha_{u u} \partial_{t}+\left(-u^{2} \alpha_{u u}+2 u \alpha_{u}-2 \alpha\right) \partial_{x^{1}}+\left(u \alpha_{u u}-\alpha_{u}\right) \partial_{x^{2}}+ \\
& \left(-\alpha_{u t}+u^{2} \alpha_{u x^{1}}-u \alpha_{u x^{2}}\right) \partial_{u},
\end{aligned}
$$

where $\alpha=\alpha\left(u, x^{1}+u^{2} t, x^{2}-u t\right)$ are arbitrary functions of the mentioned arguments. At the same time in the class of operators (17) system (19) admits a four-dimensional (maximal) invariance algebra of form (20):

$$
Y_{1}=\partial_{t}, \quad Y_{2}=\partial_{x^{1}}, \quad Y_{3}=\partial_{x^{2}}, \quad Y_{4}=t \partial_{t}+x^{1} \partial_{x^{1}}+x^{2} \partial_{x^{2}} .
$$

In applied problems of flight dynamics we managed to obtain more interesting results simultaneously as to group transformations and physical parameters (constants).

Example 2 We shall consider the longitudinal movement of aircraft, which can be described by system (21).

$$
\begin{aligned}
& \dot{h}=V \sin \theta, \quad \dot{V}=\frac{1}{m}\left(P-\left(A c_{y}^{2}+B\right) \frac{\rho V^{2}}{2} S-m g \sin \theta\right), \\
& \dot{L}=V \cos \theta, \quad \dot{\theta}=\frac{1}{m V}\left(c_{y} \frac{\rho V^{2}}{2} S-m g \cos \theta\right),
\end{aligned}
$$

where $V$ is the speed, $h$ is the altitude, $\theta$ is the angle of climb, $L$ is the distance, $(P, g, S, A$, $\left.B, c_{y}, \rho, m\right)$ are some aerodynamic coefficients.

The system (21) admits the nine-parametrical invariance algebra (22)

$$
\begin{aligned}
& X_{1}=\partial_{t}, \quad X_{2}=\partial_{h}, \quad X_{3}=\partial_{L}, \quad X_{4}=h \partial_{h}+P \partial_{P}+\rho \partial_{\rho}, \\
& X_{5}=t \partial_{t}-V \partial_{V}-2 P \partial_{P}-2 g \partial_{g}, \quad X_{6}=\rho \partial_{\rho}-S \partial_{S}, \\
& X_{7}=h \partial_{h}+L \partial_{L}+V \partial_{V}+P \partial_{P}+2 S \partial_{S}+g \partial_{g}-3 \rho \partial_{\rho}, \quad X_{8}=\partial_{A}-c_{y}^{2} \partial_{B}, \\
& X_{9}=2 h \partial_{h}+2 L \partial_{L}+t \partial_{t}+V \partial_{V}-2 c_{y} \partial_{c_{y}}+2 A \partial_{A}-2 B \partial_{B} .
\end{aligned}
$$

This fact permits to transform the system (21) by means of using invariants of operators $X_{4}-X_{9}$

$$
\begin{aligned}
& \hat{t}=t \sqrt{\frac{\rho S g\left(A c_{y}^{2}+B\right)}{2 m},} \quad \hat{h}=h \frac{\rho S}{2 m}, \quad \hat{L}=L \frac{\rho S}{2 m}, \\
& \hat{V}=V \sqrt{\frac{\rho S\left(A c_{y}^{2}+B\right)}{2 m g}}, \quad \hat{P}=\frac{P}{m g}, \quad k=\frac{c_{y}}{A c_{y}^{2}+B}
\end{aligned}
$$

to the simpler form with two parameters only ( $k$ is aerodynamic quality and $\hat{P}$ is propulsion efficiency)

$$
\begin{aligned}
& \dot{h}=V \sin \theta, \quad \dot{V}=\hat{P}-V^{2}-\sin \theta, \\
& \dot{L}=V \cos \theta, \quad \dot{\theta}=\frac{1}{V}\left(k V^{2}-\cos \theta\right)
\end{aligned}
$$


and to proceed from it to the Abel equation

$$
\frac{d E}{d \theta}(k E-\cos \theta)=2 E(P-E-\sin \theta), \quad\left(E=V^{2}\right) .
$$

If the additional condition $\left(P=V^{2}\right)$ is fulfilled, then one more operator of symmetry

$$
X_{10}=\frac{1}{V \sin \theta} \partial_{\theta}
$$

is admitted and we can construct a conservation law

$$
V \cos \theta-\int_{0}^{V} c_{y}(V) V^{2} d V=C .
$$

Thus, specific features of controlled systems make it possible (in comparison with systems of ordinary differential equations) to calculate their symmetries and to use them in applied problems of control. More detailed information can be found in [1]-[5].

\section{References}

[1] Lehen'kyj V.I., Theoretical-group algorithm in solving of the optimal control law problem, Kibernetika $i$ vychislitelnaya tekhnika (Cybernetics and computer engineering), Kyïv, 1991, V.91, 41-48.

[2] Lehen'kyj V.I., Symmetries and problem of reduction in optimal control synthesis, Kibernetika $i$ vychislitelnaya technika (Cybernetics and computer engineering), Kyïv, 1992, V.95, 12-18.

[3] Lehen'kyj V.I., Applications of Lie groups in the program control problem, Avtomatika (Automatics), Kyïv, 1992, N 6, 26-33.

[4] Legen'kyj V.I., Point symmetries and controllability of controlled systems, Dopovidi Akademï Nauk Ukrainny (Proceedings of the National Academy of Sciences of Ukraina), 1995, N 3, 15-17.

[5] Legen'kyj V.I., On the minimal-parametric form of aircraft motion's equations, International Applied Mechanics, Kyïv, 1995, N 7. 